\title{
ARTICLE OPEN \\ Local-environment dependence of stacking fault energies in concentrated solid-solution alloys
}

\author{
Shijun Zhao $\mathbb{D D}^{1,2}$, Yuri Osetsky $\mathbb{D}^{1}$, G. Malcolm Stocks ${ }^{1}$ and Yanwen Zhang $\mathbb{D}^{1,3}$
}

Concentrated solid-solution alloys (CSAs) based on 3d transition metals have demonstrated extraordinary mechanical properties and radiation resistance associated with their low stacking fault energies (SFEs). Owing to the intrinsic disorder, SFEs in CSAs exhibit distributions depending on local atomic configurations. In this work, the distribution of SFEs in equiatomic CSAs of NiCo, NiFe, and $\mathrm{NiCoCr}$ are investigated based on empirical potential and first-principles calculations. We show that the calculated distribution of SFEs in chemically disordered CSAs depends on the stacking fault area using empirical potential calculations. Based on electronic structure calculations, we find that local variations of SFEs in CSAs correlate with the charge density redistribution in the stacking fault region. We further propose a bond breaking and forming model to understand and predict the SFEs in CSAs based on the local structure alone. It is shown that the perturbation induced by a stacking fault is localized in the first-nearest planes for NiCo, but extends up to the third nearest planes for NiFe and $\mathrm{NiCoCr}$ because of partially filled $d$ electrons in $\mathrm{Fe}$ and $\mathrm{Cr}$.

npj Computational Materials (2019)5:13 ; https://doi.org/10.1038/s41524-019-0150-y

\section{INTRODUCTION}

Single-phase concentrated solid-solution alloys (CSAs) with two or more multiple principal elements situated on a simple lattice have demonstrated remarkable mechanical properties and irradiation resistance. ${ }^{1-6}$ In particular, several Ni-based face-centered cubic $(f c c)$ CSAs composed of $3 d$ transition metal elements are found to exhibit excellent fracture toughness and damage tolerance under tensile stress at cryogenic temperature. ${ }^{2,7}$ It has been shown experimentally that the high strength of CSAs is closely related to the change in deformation mechanisms from conventional dislocation glide to twining with decreasing temperature. ${ }^{2}$ On the other hand, one of the most important reasons responsible for the good radiation resistance of CSAs is the atomic-level heterogeneity, which helps to suppress dislocation movement and defect cluster growth. ${ }^{4,8}$ These results unarguably indicate that the peculiar behavior of dislocations has a significant role in governing the properties of CSAs. ${ }^{4,8-10}$ As dislocation behavior is to a large extent determined by stacking fault energies (SFEs), ${ }^{11}$ it is imperative to study the SFE properties in CSAs. A stacking fault is a planar defect in materials, and the value of the SFE represents the energy associated with interrupting the normal stacking sequence of a perfect crystal structure. In a fcc structure, the SFE also determines the dissociation distance of Shockley partial dislocations and thus the tendency of creating the local stacking fault structure (hexagonal close-packed, $h c p$ ). It is commonly assumed that deformation twinning is favored when the SFE is low, whereas dislocations slip is preferred in high SFE materials.

For chemically disordered CSAs, only limited information on SFEs is reported, partially owing to the challenges to represent the complex and vastly different atomic configurations. Theoretically, values of SFEs in some CSAs have been calculated based on firstprinciples methods, which suggest that CSAs exhibit low SFEs compared with pure $\mathrm{Ni}^{12-16}$ Experimentally, the dissociation of dislocations in NiFeCrCoMn HEAs has been observed, ${ }^{15,16}$ and the dissociation distance is found to be highly variable. It has been argued that the variations arise from fluctuations in the local chemical environment in the vicinity of a stacking fault. Indeed, in pure metals and dilute alloys, the SFE is characterized by a single value; however, in CSAs, it should be regarded as a spatially localized property that affects dislocation properties within a certain range. ${ }^{8,16}$ As the accessible stacking fault size is limited both in experimental observations and theoretical atomistic modeling, a value of SFE for CSAs estimated by any technique will depend on local environments, and variations in SFE values are related to the area considered. Therefore, the value of SFEs in CSAs exhibits a statistical distribution that originates from local disorder fluctuations statistically presented in any limited crystal size (simulation cells). Knowing the SFE distribution may help in the understanding of local dislocation behavior as revealed experimentally. More importantly, the relation between a certain SFE and its local environment is of particular interest as it can predict stability of local structures and/or deformation mechanisms. Such a relationship should also help to understand local dislocation properties in CSAs when short-range order develops or inhomogeneous composition is present, as already found in some CSAs. ${ }^{17,18}$ The established dependence of SFEs on local chemical environment may also provide guidance for tuning alloy properties of CSAs by tailoring their chemical fluctuations.

In this work, we report distributions of SFEs in equiatomic CSAs of $\mathrm{NiCo}, \mathrm{NiFe}$, and $\mathrm{NiCoCr}$ using static simulations based on both empirical potential and first-principles density functional theory (DFT) calculations. Both approaches rely on supercell models. The above systems are chosen as being typical concentrated alloys that include different combinations from several metallurgical important elements including $\mathrm{Ni}, \mathrm{Co}, \mathrm{Fe}$, and $\mathrm{Cr}$. In addition, these elements are $3 d$ transition metals with different electronic

\footnotetext{
${ }^{1}$ Materials Science and Technology Division, Oak Ridge National Laboratory, Oak Ridge, TN 37831, USA; ${ }^{2}$ Department of Mechanical Engineering, City University of Hong Kong, Hong Kong, China and ${ }^{3}$ Department of Materials Science and Engineering, University of Tennessee, Knoxville, TN 37996, USA

Correspondence: Shijun Zhao (shijzhao@cityu.edu.hk)
}

Received: 6 August 2018 Accepted: 28 December 2018

Published online: 04 February 2019 
structures, in which $\mathrm{Ni}$ and Co have nearly full-filled $d$ electrons, whereas $\mathrm{Fe}$ and $\mathrm{Cr}$ possess only partially filled $d$ electrons. These elements are also the base elements that appear in many other complex concentrated alloys (e.g., high entropy alloys). The dependence of SFE distribution on the stacking fault area is firstly illustrated by empirical potential calculations. Afterwards, the local environment dependence of SFE under different local structures is analyzed based on DFT results. It is found that the distribution of SFEs in CSAs correlates with the charge density redistribution in the stacking fault region. Based on electronic structure results, we propose a bond breaking and forming model to understand the SFE distributions in CSAs.

\section{RESULTS AND DISCUSSION}

We first show the dependence of SFE distribution in CSAs on the stacking fault area. For this purpose, the SFE distribution in equiatomic random NiFe alloys is calculated for different stacking fault areas with an empirical interatomic potential. ${ }^{19}$ Note that this potential is optimized specifically for the description of SFE in austenitic stainless steels. In pure $\mathrm{Ni}$, it results in a SFE value of $130 \mathrm{~mJ} / \mathrm{m}^{2}$, which is in good agreement with the value of $133 \mathrm{~mJ} / \mathrm{m}^{2}$ from our first-principles calculations. The SFE distributions in NiFe calculated for different stacking fault areas are presented in Fig. 1. At each stacking fault size, 3000 stacking fault configurations are simulated by randomly distributing $\mathrm{Ni}$ and $\mathrm{Fe}$ atoms on the lattice. Figure $1 \mathrm{a}$ illustrates the very broad distribution of SFE, ranging from -80 to $+140 \mathrm{~mJ} / \mathrm{m}^{2}$, for the
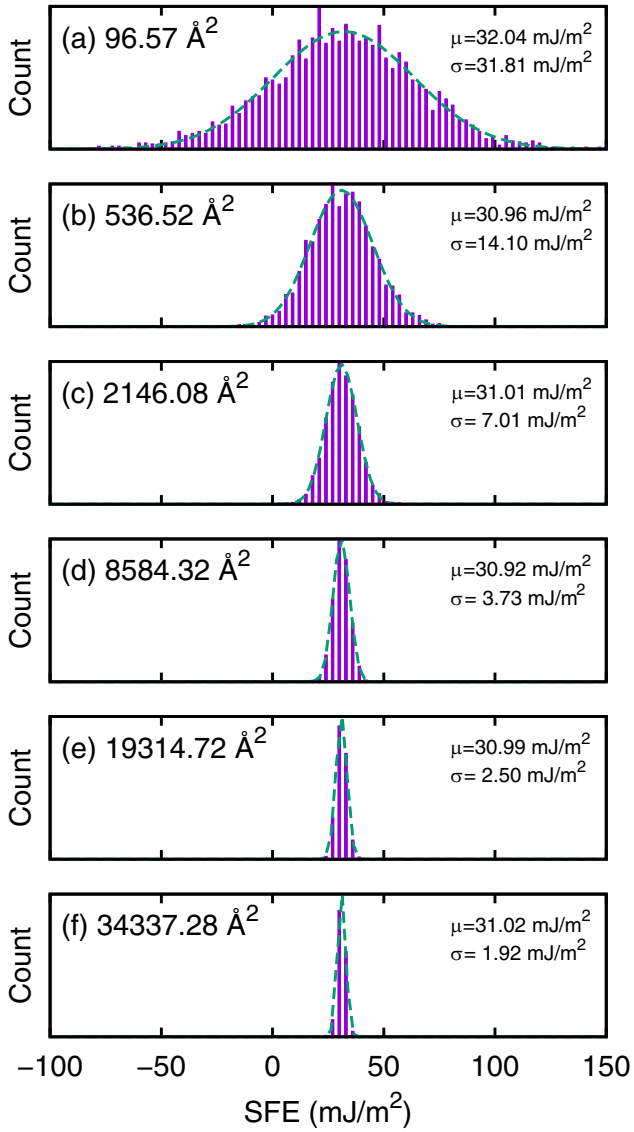

Fig. 1 Distributions of SFE in NiFe calculated from supercells with 60 [1111] planes. In each case, 3000 stacking fault configurations with different $\mathrm{Ni}$ and Fe distributions are simulated. The stacking fault area in the [112]-[ī10] plane in each case is indicated. A Gaussian distribution function is fitted to these distributions and the obtained mean value $\mu$ and standard deviation $\sigma$ are provided smallest stacking fault area studied. As the area of the stacking fault increases, the distribution of SFE narrows. These SFE distributions are fitted with a Gaussian distribution, which yields the mean value $\mu$ and standard deviation $\sigma$, as given in Fig. 1 . From the fitted results, it is clear that $\sigma$ decreases rapidly with increasing stacking fault area. Indeed, $\sigma$ is found to scale inversely with the square root of the area $(\sigma \sim 1 / \sqrt{A})$ owing to the mean field effect in combination with the random distribution of elements. In the limit of infinite stacking fault area, $\sigma$ will become zero and the SFE is a single value. Nevertheless, the mean SFE value is not much influenced by the stacking fault area, suggesting a possibility of estimating the SFE limit for an infinite stacking fault area using a finite supercell as long as the statistical sampling is large enough. Based on the potential used here, the mean SFE value in NiFe is $\sim 31 \mathrm{~mJ} / \mathrm{m}^{2}$.

The dependence of the SFE distribution on the stacking fault area indicates that SFE depends strongly on local atomic environment, thus the SFE should be regarded as a local quantity. This is in agreement with experimental observations, which show large fluctuations of SFEs related to the local chemical environments in the vicinity of the stacking faults. ${ }^{16}$ To reveal the local environment effect on SFE values, we have carried out a series of first-principles calculations to study SFE distributions in three model CSAs of $\mathrm{NiCo}, \mathrm{NiFe}$, and $\mathrm{NiCoCr}$. The SFE values obtained are analyzed with respect to local atomic environment in the stacking fault region.

In pure metals and dilute alloys, variations of SFE and theoretical shear strength are often analyzed qualitatively in terms of charge density redistributions and perturbation of bond strength. ${ }^{20-22}$ It should be noted that the influence of stacking fault is generally localized within the stacking fault region, as verified by previous first-principles calculations in $f c c$ Fe. ${ }^{23}$ Based on this consideration, a quantitative relation between SFE and maximum value of charge difference on the stacking fault plane has been proposed previously to explain SFE changes in different fcc materials. ${ }^{24}$ However, in CSAs where different atoms all at high concentrations are randomly distributed in the stacking fault region, the charge redistribution induced by a stacking fault is similar in different configurations, especially when elemental concentrations near the stacking fault are close. An example is illustrated in Figure S1 in Supplementary Information, where the charge redistribution in the central stacking fault plane is provided for NiFe with significantly different SFE values. The charge difference plot shows that, regardless of the elemental concentration in the first-nearest neighbor planes (1nnp), the maximal charge transfer is similar in all three cases. Thus, the maximal charge difference alone is not appropriate for understanding SFE distributions in CSAs, and we need to explore the details of charge redistribution instead.

To this end, we introduce a new physical quantity to describe the charge density redistribution $(\Delta \rho)$ in the stacking fault region. Here $\Delta \rho$ is defined by the charge variation at bond critical points (cps), which are the points with zero gradient of charge density along the bond direction:

$\Delta \rho=\frac{1}{n_{b}} \sum_{b i}\left[\rho_{i}(S F)-\rho_{i}(\right.$ per $\left.)\right]$,

where $n_{b}$ is the number of bonds in the stacking fault region, $\rho_{i}$ (SF) and $\rho_{i}$ (per) are the charge density at bond cps in the stacking fault and perfect structures, respectively. All the first-nearest neighbor chemical bonds within the stacking fault region are considered. As shown in Fig. 2, the averaged $\Delta \rho$ at all cps correlates well with the calculated SFE values. This result provides the first evidence that $\Delta \rho$ may be used to estimate SFE values in a specific atomic environment, and thus local SFE in concentrated alloys can be understood in terms of the local atomic environment. Previously, bond cps were used to understand different 

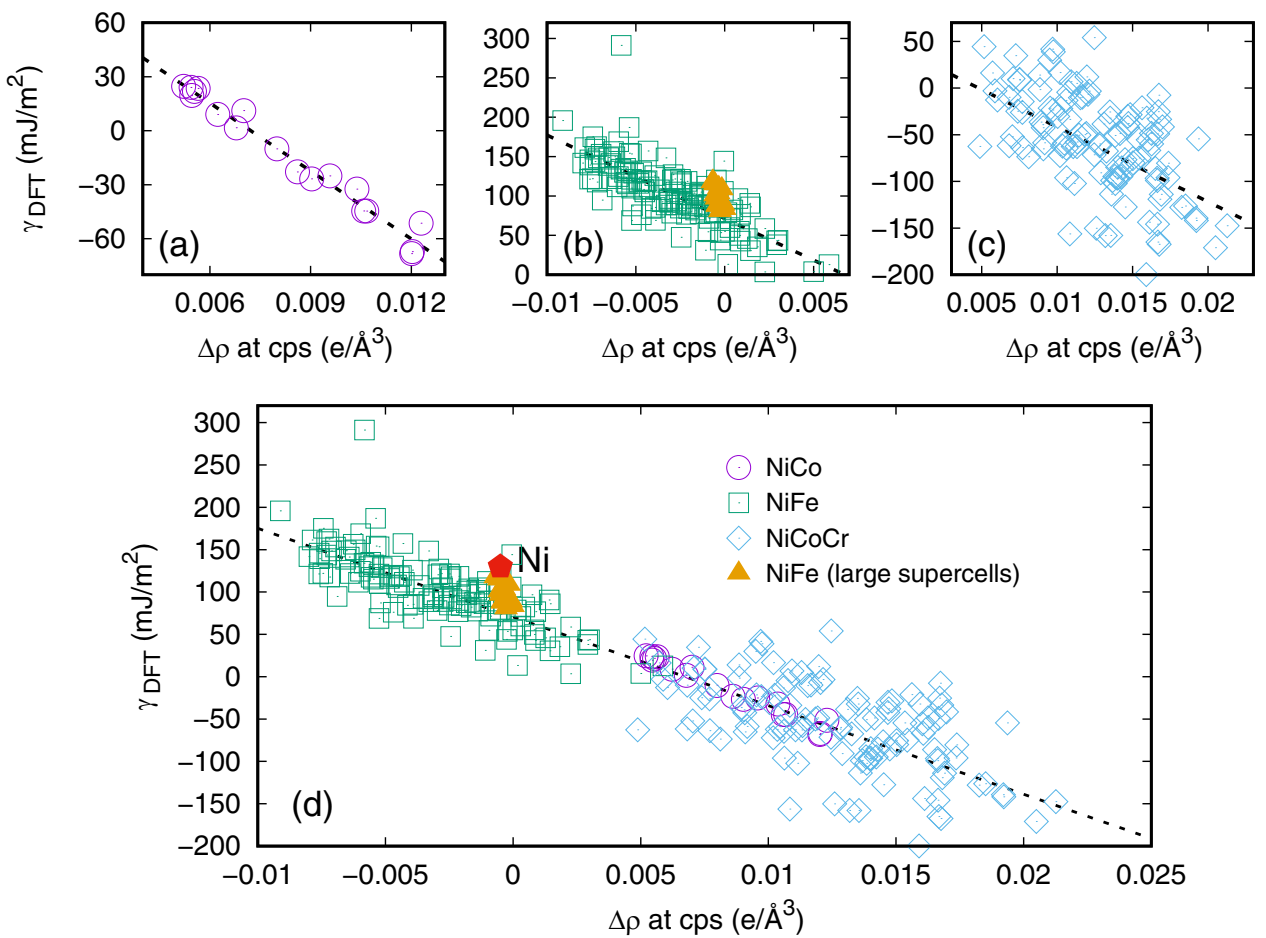

Fig. 2 Calculated SFE values with respect to charge redistribution at bond critical points (cps) in the stacking fault region: a NiCo, b NiFe, c $\mathrm{NiCoCr}$, and $\mathbf{d}$ all the data points from our calculations. The dashed line is a linear fitting of the data. For validation purpose, another 15 results in NiFe from larger supercell calculations are included. The results obtained in pure $\mathrm{Ni}$ is also indicated in $\mathbf{d}$

generalized stacking fault energy curves ( $\gamma$ surface) in different $f c c$ metals. ${ }^{25,26}$ However, no quantitative connection between charge density and SFEs was established. The correlation between our proposed $\Delta \rho$ and local SFE values is consistent in all three considered CSAs. In particular, for pure $\mathrm{Ni}, \Delta \rho=-0.005 \mathrm{e} / \AA^{3}$ and $\gamma$ $=130 \mathrm{~mJ} / \mathrm{m}^{2}$, which is in line with the results in Fig. 2 . The results in Fig. 2 are obtained within a relatively small supercell $(2 \times$ 3 supercell in the $x-y$ plane). In order to further prove and validate our results, we have carried out additional calculations for $\mathrm{NiFe}$ using a larger $3 \times 5$ supercell. As our results show that the contribution to local stacking fault energy from the atomic relaxation is small (as can be seen from Figure S2 in Supplementary Information), we do not relax atomic positions in the large supercell calculations. The obtained results are also included in Fig. 2, which show that the correlation between $\Delta \rho$ and local SFE values is consistent for all calculations. The calculated SFEs in these large supercells are concentrated in a smaller range as more local environments are sampled and the effects are averaged, as expected from Fig. 1. A larger $\Delta \rho$ indicates a higher charge density in the stacking fault structure $(h c p)$. As a result, a large $\Delta \rho$ suggests that the stability of the original fcc structure over the $h c p$ configurations decreases, which leads to a low SFE. In NiCoCr, a very large $\Delta \rho$ is observed, indicating that the $h c p$ structures are energetically preferred over $f c c$, in agreement with our energy difference calculations. ${ }^{13}$

In Fig. 2, it is shown that the SFE distribution in NiCo is relatively narrow compared with that in $\mathrm{NiFe}$ and $\mathrm{NiCoCr}$. For reference, the SFE values are -120.61 and $-465.65 \mathrm{~mJ} / \mathrm{m}^{2}$ for pure ferromagnetic fcc $\mathrm{Co}$ and $\mathrm{Fe}$, respectively. To probe the effect of atomic relaxation on SFEs, we have calculated SFEs in NiFe without relaxation in selected cases. The results are provided in Figure S2 in Supplementary Information, which makes an important point that relaxation leads to only minor modification of calculated SFEs. Therefore, the observed SFE distribution is mainly attributed to electronic interactions. Further evidence for the large variation of SFE values in NiFe compared with NiCo can be found from the evolution of local magnetic moments of atoms in $1 \mathrm{nnp}, 2 \mathrm{nnp}$, and $3 \mathrm{nnp}$ around the stacking fault, as shown in Fig. 3 , along with the calculated SFE values for each structure. The relaxed local magnetic moments were determined by integrating the spin density within spheres centered on the atoms. ${ }^{25}$ We have checked the variation of the volume of atomic spheres between the perfect and stacking fault structures. As both structures are close-packed, their atomic sphere volumes are only slightly different. With a Voronoi tessellation method, ${ }^{27}$ we find that the volume change does not exceed $0.5 \%$, which indicates the noise would be $<0.5 \%$ in integrated moments. This value is almost the same order of moment changes in $3 \mathrm{nn}$. It is seen that the magnetic perturbations mainly occur in the atoms located at $1 \mathrm{nnp}$ and $2 \mathrm{nnp}$, whereas the atoms located far away from the stacking fault are weakly affected. Thus, it is mainly the atoms near the stacking fault that significantly contribute to SFE values. Although the moments of $\mathrm{Ni}$ atoms $\left(\Delta \mu_{\mathrm{Ni}}\right)$ in $1 \mathrm{nnp}$ increase after the introduction of a stacking fault in both NiCo and NiFe alloys, the changes of Co and Fe moments $\left(\Delta \mu_{\mathrm{Co}}\right.$ and $\left.\Delta \mu_{\mathrm{Fe}}\right)$ are different. Nevertheless, a clear tendency can still be identified in NiCo, in which a high $\Delta \mu_{\mathrm{Ni}}$ combined with a low $\Delta \mu_{\text {co }}$ leads to a low SFE value, whereas a low $\Delta \mu_{\mathrm{Ni}}$ and a high $\Delta \mu_{\mathrm{Co}}$ results in a high SFE value. This phenomenon is also observed for atoms located at $2 \mathrm{nnp}$. However, in the NiFe alloy, no clear correlation is found between the magnetic moment variation and the SFE value. The evolution of magnetic moments of $\mathrm{Fe}$ in $\mathrm{NiFe}$ is more susceptible to the surrounding environment. Notably, the moment change is much larger in NiFe at $2 \mathrm{nnp}$ and $3 \mathrm{nnp}$, suggesting that a stacking fault in NiFe has a long-range effect compared with that in NiCo.

This finding is consistent with the results of electronic density of states (DOS) calculated in the perfect and stacking fault structures. In Fig. 4, the DOS of atoms in $1 \mathrm{nnp}, 2 \mathrm{nnp}$, and 3nnp in structures with the highest and lowest SFE are given for both NiCo and NiFe. For these transition metals, their total DOS is dominated by $d$ electrons. In $\mathrm{NiFe}$, the calculated DOS in structures with the highest SFE shows a significant increase below the Fermi level 

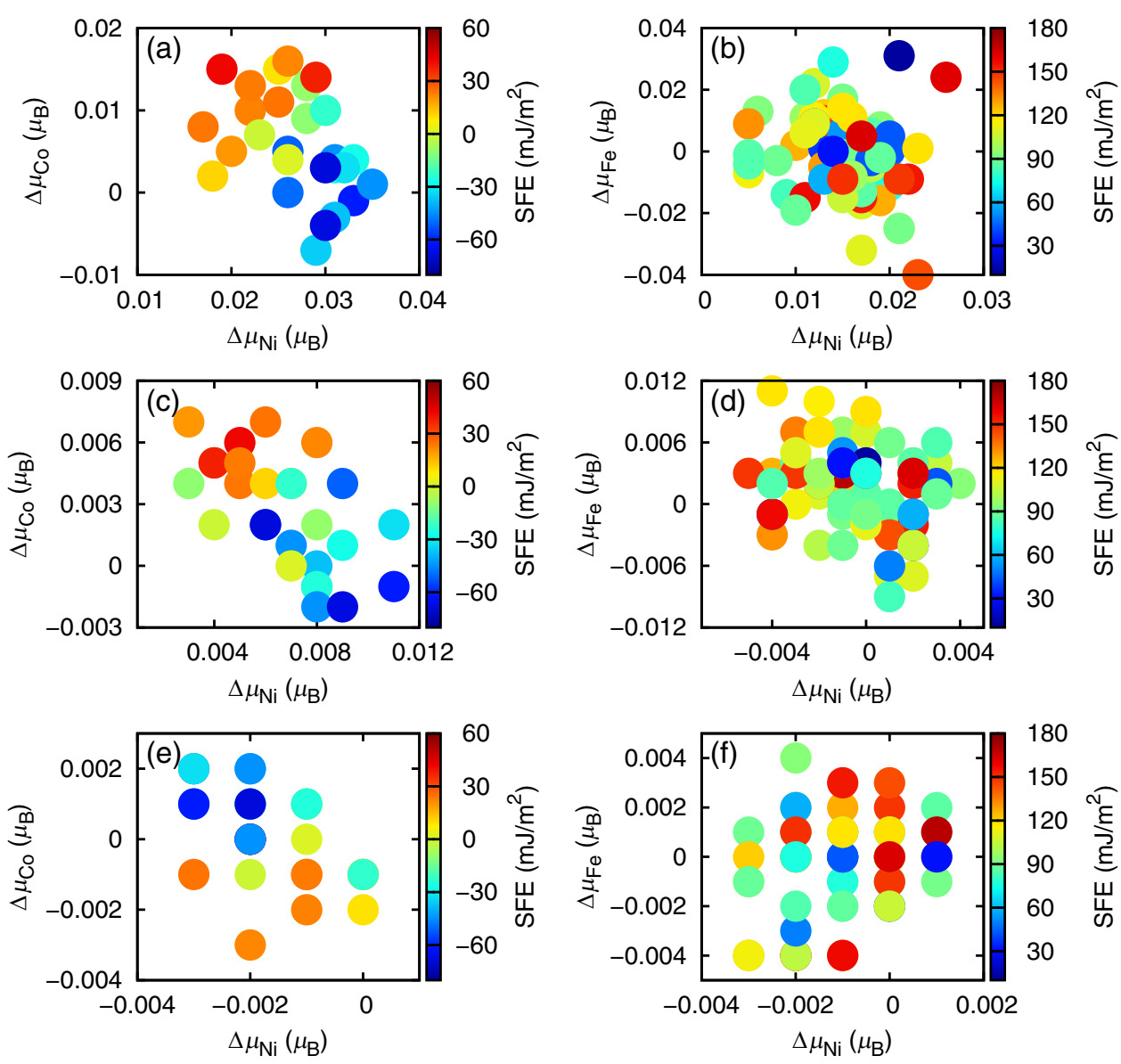

Fig. 3 Variation of magnetic moment associated with calculated SFE in NiCo and NiFe. The magnetic moments are averaged from atoms in $1 \mathrm{nnp}$ (a and b), 2 nnp (c and $\mathbf{d})$, and 3nnp (e and $\mathbf{f}$ )

even in the $2 \mathrm{nnp}$. This observation indicates an increasing band filling in this case, which contributes to a higher band energy. On the other hand, in the structures with the lowest SFE, band filling decreases mainly in $1 \mathrm{nnp}$, which contributes to the low SFE. These results suggest that SFE is dominated by the band energy. The stacking fault induces changes beyond $1 \mathrm{nnp}$ in NiFe and $\mathrm{NiCoCr}$, which can further be seen from occupation changes of $d$ electrons, as given in Figure S3 in Supplementary Information. In $\mathrm{NiCoCr}$, the occupation of $d$ electrons for atoms in $3 \mathrm{nnp}$ still shows significant perturbation. This can be ascribed to partially filled $d$ electrons in $\mathrm{Fe}$ and $\mathrm{Cr}$, which provide electron density flexibility in the presence of defects such as point defects ${ }^{28}$ and stacking faults. As the main contribution to $\Delta \rho$ is from atoms near the stacking fault region, its prediction is less accurate for $\mathrm{NiFe}$ and $\mathrm{NiCoCr}$ as displayed in Fig. 2.

The above discussion suggests that the distribution of SFEs in CSAs can be analyzed in terms of the local atomic environment. To achieve this, we propose a bond breaking and forming model to estimate local SFEs based on the local structure alone. In a fcc structure, every atom in a [111] plane has three nearest neighbors $(n n)$ in the adjacent [111] planes, forming three atomic interlayer bonds. When a shear deformation starts and finally creates a stacking fault structure, one of these three bonds breaks and another new bond is established, maintaining the three nearest neighbor configuration between adjacent layers as the resulting $h c p$ structure is also a close-packed structure. In this process, every atom is associated with an old bond breaking and a new bond forming. Such bond breaking and forming events contribute most to the $\Delta \rho$, and thus are the dominant contributions to SFE. Therefore, SFE can be regarded as a measure of the total contribution from these energy terms:

$\gamma=\sum_{i} J_{i} n_{i}$,

where $J_{i}$ is the energy contribution from a given bond breaking and forming event and $n_{i}$ is the number of this bond breaking and forming type, respectively. For example, there is 1 type of bond forming and breaking in pure $\mathrm{Ni}(\mathrm{Ni}-\mathrm{Ni} \rightarrow \mathrm{Ni}-\mathrm{Ni})$, seven types in binary alloys (e.g., Ni-Ni $\rightarrow \mathrm{Ni}-\mathrm{Ni}, \mathrm{Ni}-\mathrm{Ni} \rightarrow \mathrm{Ni}-\mathrm{Fe}, \mathrm{Ni}-\mathrm{Fe} \rightarrow \mathrm{Ni}-\mathrm{Ni}, \mathrm{Ni}-$ $\mathrm{Fe} \rightarrow \mathrm{Ni}-\mathrm{Fe}, \mathrm{Ni}-\mathrm{Fe} \rightarrow \mathrm{Fe}-\mathrm{Fe}, \mathrm{Fe}-\mathrm{Fe} \rightarrow \mathrm{Ni}-\mathrm{Fe}$, and $\mathrm{Fe}-\mathrm{Fe} \rightarrow \mathrm{Fe}-\mathrm{Fe}$ in $\mathrm{NiFe})$, and 24 types in ternary $\mathrm{NiCoCr}$ alloys, as detailed in Table 1 together with the obtained $J_{i}$ from the fitting based on Eq. (2). Following this procedure, the calculated SFEs from DFT $\left(\gamma_{\mathrm{DFT}}\right)$ and those from Eq. $2\left(\gamma_{\mathrm{FIT}}\right)$ are presented in Fig. 5 . It can be seen that the model captures the general feature of the local environment dependence of local SFEs in CSAs.

Based on the results of $J_{i}$ shown in Table 1 , it can be seen that $\mathrm{Ni}-\mathrm{Ni} \rightarrow \mathrm{Ni}-\mathrm{Co}$ events have the highest contribution of $19.26 \mathrm{~mJ} /$ $\mathrm{m}^{2}$ to SFE of NiCo, whereas the contribution from Co-Co- $>\mathrm{Ni}-\mathrm{Co}$ is the lowest of $-21.53 \mathrm{~mJ} / \mathrm{m}^{2}$. Therefore, the Co-Co bond is unstable in fcc NiCo and it is easier to be broken than $\mathrm{Ni}-\mathrm{Ni}$. Similarly, Fe-Fe bond is also easier to be broken in fcc NiFe. These results are in line with the force constant calculations in these disordered alloys, which show weaker interactions in Co-Co (Fe-Fe) compared with in Ni-Ni.

For the case of $\mathrm{NiCo}$, the strong correlations shown in Figs 2 and 3 suggest that it should be possible to model SFEs in NiCo directly based on local elemental concentrations near the stacking fault region. In Fig. 6a, the SFE values are given with respect to the concentration of $\mathrm{Ni}$ and $\mathrm{Co}$ in the $1 \mathrm{nnp}$ around the stacking fault. 

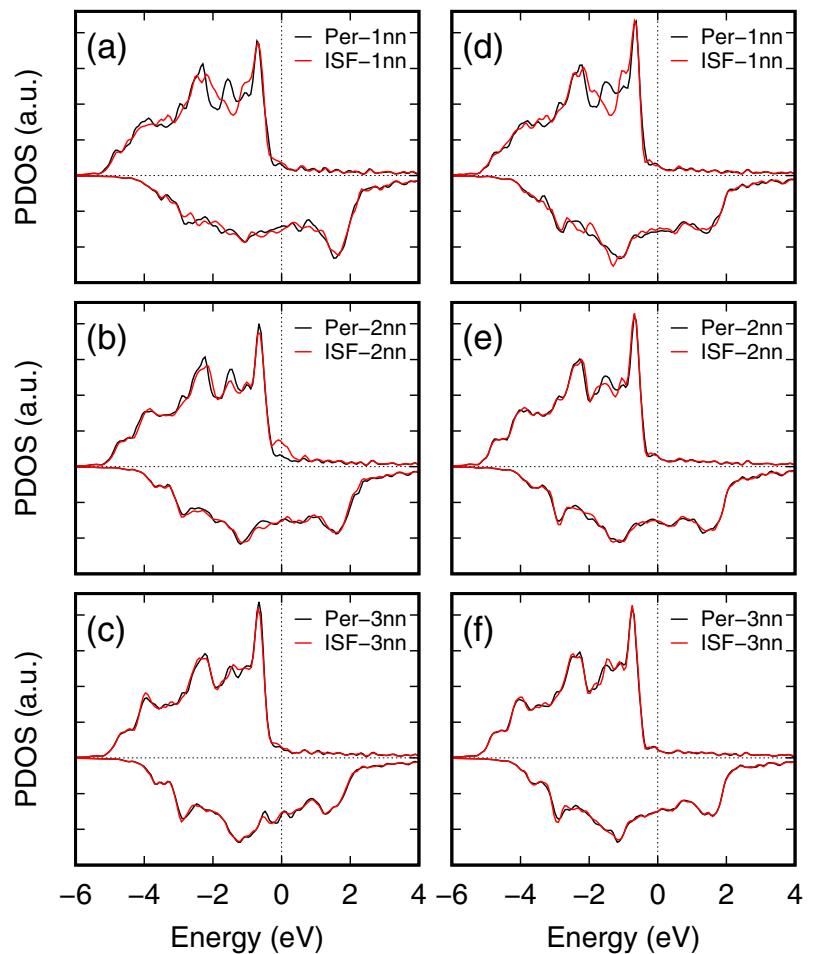

Fig. 4 Evolution of projected density of states for $d$ electrons in the stacking fault region for NiFe after the introduction of a stacking fault. Two cases are given: the highest SFE (left column) and the lowest SFE (right column)

A high concentration of $\mathrm{Ni}$ leads to a high SFE value, whereas a high concentration of Co results in a low or even negative SFE value in NiCo because the ground state of $\mathrm{Co}$ is $h c p$. As the concentrations of $\mathrm{Ni}$ and $\mathrm{Co}$ are not independent, the SFE values can be written as $\gamma=\sum_{i=1}^{3} A_{i} C_{i}^{N i}+A_{0}$, where $A_{i}$ is the interaction contribution from $\mathrm{Ni}$ in the nearest $i$ plane from the stacking fault and $A_{O}$ is a constant. Here, only the first three nnp are considered. The results $\left(\gamma_{\mathrm{Fit}}\right)$ are presented in Fig. $6 \mathrm{~b}$, which reproduce the DFT data very well, indicating that consideration of the first three nnp is sufficient to describe the SFE distribution in NiCo. The correlation coefficients $\rho$ is 0.99 for this regression. As discussed above, although there is strong correlation between $\Delta \rho, \Delta \mu$, and the local stacking fault energies in $\mathrm{NiCo}$, these correlations are weaker in NiFe or $\mathrm{NiCoCr}$. As a result, it is difficult to establish a linear relation between SFE and local concentrations in NiFe and $\mathrm{NiCoCr}$ (particularly the latter) as a result of charge and magnetization rearrangements in the neighborhood of the stacking fault. Hence, it is not possible to establish a direct relation between local SFEs and local concentrations in NiFe and $\mathrm{NiCoCr}$. For these alloys, it is therefore necessary to consider the contribution to the local SFE from every atom, as each $\mathrm{Fe}$ and $\mathrm{Cr}$ behaves differently in response to its unique local environment.

The distribution of SFE depends on stacking fault area considered. In our DFT calculations, relatively small supercells are used, which yield large fluctuations of SFEs. As we are focused on the local environment dependence of SFEs, the same supercell size is employed for all three alloys so as to describe such dependence by developing a unified model. Our results show that a bond breaking and formation model is appropriate to capture the general trend of local structure dependence of SFEs in concentrated alloys, even for the small stacking fault size considered. Our calculated SFEs at $0 \mathrm{~K}$ exhibit some negative values, especially for $\mathrm{NiCoCr}$. This is in agreement with previous
Table 1. The energy contribution $\left(J_{i}\right.$, in $\left.\mathrm{mJ} / \mathrm{m}^{2}\right)$ from a given bond breaking and forming type in CSAs investigated

\begin{tabular}{|c|c|c|c|c|c|}
\hline index & Bond change & $\mathrm{NiCo}$ & NiFe & Bond change & $\mathrm{NiCoCr}$ \\
\hline 1 & $11 \rightarrow 11$ & 11.20 & 3.04 & $11 \rightarrow 11$ & 1.10 \\
\hline 2 & $11 \rightarrow 12$ & 19.26 & 15.83 & $11 \rightarrow 12$ & 5.11 \\
\hline 3 & $12 \rightarrow 11$ & -6.63 & -5.41 & $11 \rightarrow 13$ & 10.10 \\
\hline 4 & $12 \rightarrow 12(21 \rightarrow 21)$ & -2.18 & 6.27 & $12 \rightarrow 11$ & 7.82 \\
\hline 5 & $21 \rightarrow 22$ & 7.31 & 40.48 & $12 \rightarrow 12(21 \rightarrow 21)$ & -3.32 \\
\hline 6 & $22 \rightarrow 21$ & -21.53 & -17.47 & $12 \rightarrow 13$ & -3.95 \\
\hline 7 & $22 \rightarrow 22$ & -12.64 & 20.65 & $13 \rightarrow 11$ & -5.47 \\
\hline 8 & & & & $13 \rightarrow 12$ & 3.73 \\
\hline 9 & & & & $13 \rightarrow 13(31 \rightarrow 31)$ & 0.33 \\
\hline 10 & & & & $21 \rightarrow 22$ & -20.29 \\
\hline 11 & & & & $21 \rightarrow 23$ & 5.48 \\
\hline 12 & & & & $22 \rightarrow 21$ & -4.68 \\
\hline 13 & & & & $22 \rightarrow 22$ & -5.02 \\
\hline 14 & & & & $22 \rightarrow 23$ & -15.78 \\
\hline 15 & & & & $23 \rightarrow 21$ & -8.10 \\
\hline 16 & & & & $23 \rightarrow 22$ & -3.20 \\
\hline 17 & & & & $23 \rightarrow 23(32 \rightarrow 32)$ & -10.68 \\
\hline 18 & & & & $31 \rightarrow 32$ & -12.69 \\
\hline 19 & & & & $31 \rightarrow 33$ & 21.81 \\
\hline 20 & & & & $32 \rightarrow 31$ & -4.76 \\
\hline 21 & & & & $32 \rightarrow 33$ & -0.81 \\
\hline 22 & & & & $33 \rightarrow 31$ & -40.03 \\
\hline 23 & & & & $33 \rightarrow 32$ & -27.34 \\
\hline 24 & & & & $33 \rightarrow 33$ & -19.46 \\
\hline
\end{tabular}

calculations. ${ }^{13}$ By including contributions from other factors, such as vibrational entropy and magnetic entropy, SFEs at finite temperature can be calculated and compared well with experiments. ${ }^{12,13}$ Nevertheless, thermal effects are more general and are applied to the whole system. The main contribution of local environment dependence of SFE comes from random arrangement of elements. In this study, we focus on local SFEs in CSAs, which is the first step toward understanding unusual dislocation properties in CSAs.

In summary, because of local chemical disorder (random elemental arrangement), SFE values in CSAs exhibit distributions depending on the size of the stacking fault. We find the influence of a stacking fault is local in NiCo ( 1nnp), but relatively longrange in NiFe and NiCoCr ( 3nnp). Based on electronic structure analysis, we establish the first evidence that local SFE in CSAs can be characterized by the charge density redistribution at bond critical points. We further propose a bond breaking and forming model that only considers the short-range effect to predict the local SFE in a specific atomic configuration. Our model captures the general feature of the dependence of the SFE on local environment in concentrated alloys containing typical metallurgically useful elements. These results establish a clear link between local atomic arrangement and the corresponding SFEs, which are important for understanding the local properties of CSAs and predicting the associated effects (e.g., phase and structural stability, atomic transport, dislocation properties, etc), where local property fluctuations may play a crucial role, such as initiation of particular modes of plastic deformation. 

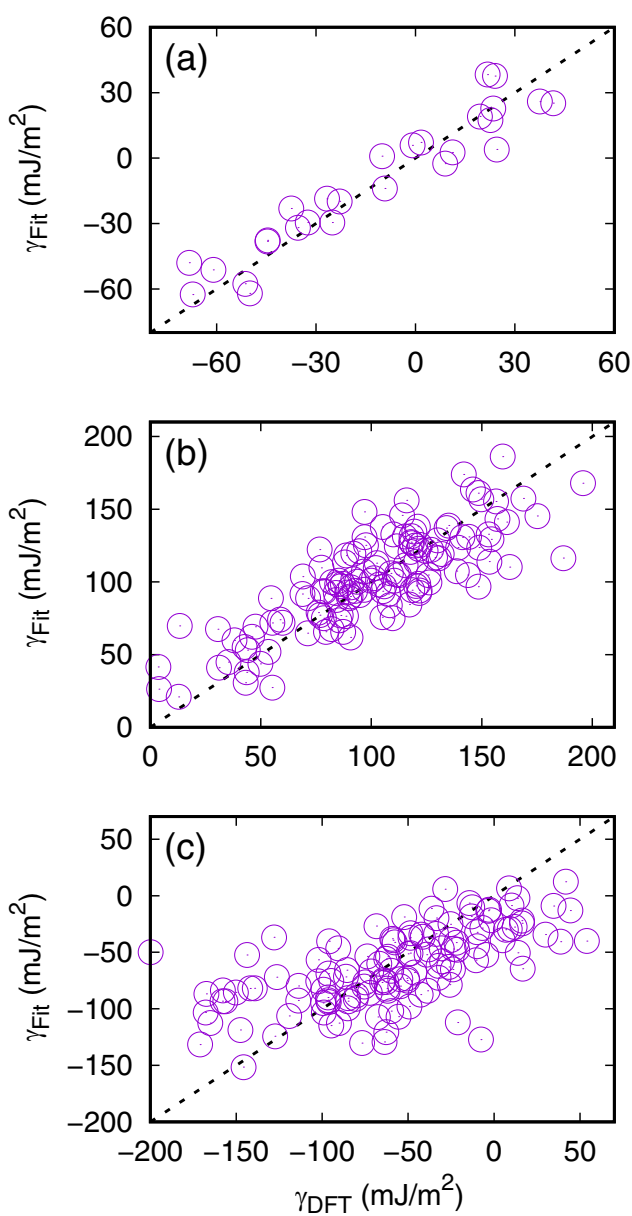

Fig. 5 SFE from DFT calculations and fitted by a bond breaking and forming model. a NiCo, b NiFe, and c NiCoCr

\section{METHODS}

First-principles calculations were based on DFT as implemented in the Vienna ab initio simulation package (VASP). ${ }^{29}$ A gradient corrected functional in the Perdew-Burke-Ernzerhof form was used to describe the exchange and correlation interactions. ${ }^{30}$ Electron-ion interactions were treated within the projector-augmented-wave PAW method. ${ }^{31}$ The energy cutoff for the plane-wave basis set was set to be $270 \mathrm{eV}$ and the energy convergence was set to be $10^{-6} \mathrm{eV}$. Standard PAW pseudopotentials distributed with VASP were adopted. All calculations were performed with spin-polarization to account for the magnetic properties of considered alloys. It turns out that $\mathrm{Ni}, \mathrm{Co}$, and Fe sites are coupled ferromagnetically with themselves and each other, whereas $\mathrm{Cr}$ sites prefer to couple antiferromagnetically with other $\mathrm{Cr}$ sites as well as $\mathrm{Ni}, \mathrm{Co}$, and Fe sites.

The chemical disorder of the CSAs was considered using special quasirandom structures (SQS), which was constructed by optimization of the Warren-Cowley short-range order parameters ${ }^{32,33}$ through a simulated Monte-Carlo algorithm. ${ }^{13}$ A SQS with 108 atoms oriented in the [112], [1110] and [ $\overline{1} \overline{1} 1]$ directions consisting of 9 [ [1 $\overline{1} 1]$ planes was constructed by considering 3D periodical boundary conditions. After the SQS structure was obtained, a vacuum layer with a thickness of larger than $10 \AA$ was added and an intrinsic stacking fault (ISF) was introduced by rigidly shifting the upper four [ $\overline{1} \overline{1} 1]$ layers in the [112] direction with respect to the lower five [ $\overline{1} \overline{1} 1]$ layers. This process was repeated for every possible position of stacking fault and the stacking sequence was manually changed in order to ensure the stacking fault was always located in the middle of the supercell. The Brillouin zone was sampled using 「-point-based $4 \times 4 \times 2$ mesh. For each rigid shift, the atomic positions were relaxed along the close-packed direction by minimizing the Hellmann-Feynman forces on each atom to $<10^{-2} \mathrm{eV} / \AA \AA$. Note that for the long vacuum distance used here, one $k$-point is enough to converge the total energy. However, we choose to increase the $k$-point in this direction to improve accuracy in SFE calculations where relaxation processes are allowed only along the vacuum
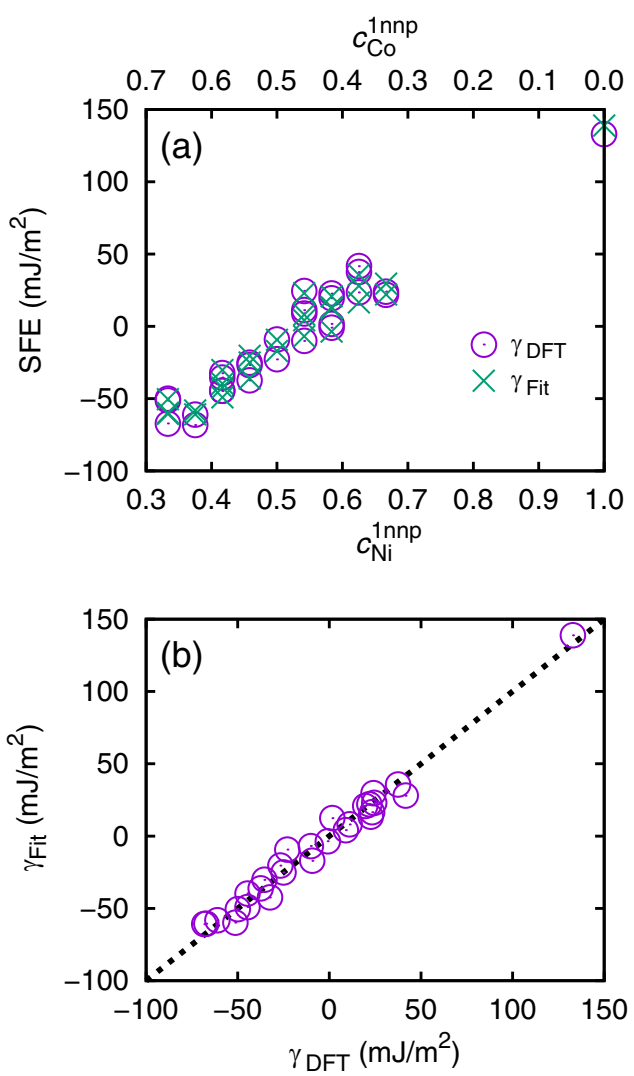

Fig. 6 a Dependence of SFE in NiCo on the local concentration of $\mathrm{Ni}$ and $\mathrm{Co}$ in the first-nearest neighbor planes (1nnp). b SFE in NiCo obtained from DFT $\left(\gamma_{\mathrm{DFT}}\right)$ compared with the results estimated from linear regression $\left(\gamma_{\mathrm{Fit}}\right)$. The correlation coefficient is 0.99 between these two sets of SFE

direction. The SFE was calculated by the formula:

$\gamma_{I S F}=\frac{1}{A}\left(E_{I S F}-E_{0}\right)$

where $A$ is the area of ISF, $E_{I S F}$ and $E_{0}$ is the energy of the configurations with and without ISF, respectively. In order to obtain the distribution of SFE, different SQS supercells were used and different SFE values were calculated from different stacking fault structures. For reference, the SFE values are $132.78,-120.61$, and $-465.65 \mathrm{~mJ} / \mathrm{m}^{2}$ for pure ferromagnetic $\mathrm{fcc}$ $\mathrm{Ni}, \mathrm{Co}$, and $\mathrm{Fe}$, respectively.

Molecular dynamics simulations were performed using the large-scale atomic/molecular massively parallel simulator. ${ }^{34}$ The interatomic potentials developed by Bonny et al. ${ }^{19}$ based on the embedded atom method were

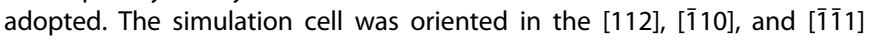
directions, which included 60 [ $\overline{1} \overline{1} 1]$ planes with different size in the other two directions. The stacking fault is introduced in the center of the supercell to calculate SFE.

\section{DATA AVAILABILITY}

The authors declare that the data supporting the findings of this study are available within the paper and supplement. Also, the data that support the plots within this paper and other findings of this study are available from the corresponding author upon reasonable request.

\section{ACKNOWLEDGEMENTS}

This work was supported as part of the Energy Dissipation to Defect Evolution (EDDE), an Energy Frontier Research Center funded by the US Department of Energy, Office of Science, Basic Energy Sciences under contract number DE-AC05-00OR22725. The calculations during revision stage was supported by City University of Hong Kong. 


\section{AUTHOR CONTRIBUTIONS}

S. Zhao performed the calculations and drafted the initial manuscript. All authors contributed to the manuscript writing.

\section{ADDITIONAL INFORMATION}

Supplementary information accompanies the paper on the npj Computational Materials website (https://doi.org/10.1038/s41524-019-0150-y).

Competing interests: The authors declare no competing Interests.

Publisher's note: Springer Nature remains neutral with regard to jurisdictional claims in published maps and institutional affiliations.

\section{REFERENCES}

1. Cantor, B., Chang, I. T. H., Knight, P. \& Vincent, A. J. B. Microstructural development in equiatomic multicomponent alloys. Mater. Sci. Eng. A 375, 213-218 (2004)

2. Gludovatz, B. et al. A fracture-resistant high-entropy alloy for cryogenic applications. Science 345, 1153-1158 (2014).

3. Yeh, J.-W. et al. Nanostructured high-entropy alloys with multiple principal elements: novel alloy design concepts and outcomes. Adv. Eng. Mater. 6, 299-303 (2004).

4. Zhang, Y. et al. Atomic-level heterogeneity and defect dynamics in concentrated solid-solution alloys. Curr. Opin. Solid State Mater. Sci. 21, 221-237 (2017).

5. Zhao, S., Weber, W. J. \& Zhang, Y. Unique challenges for modeling defect dynamics in concentrated solid-solution alloys. JOM 69, 2084-2091 (2017).

6. Zhao, S. et al. Suppression of vacancy cluster growth in concentrated solid solution alloys. Acta Mater. 125, 231-237 (2017).

7. Gludovatz, B. et al. Exceptional damage-tolerance of a medium-entropy alloy CrCoNi at cryogenic temperatures. Nat. Commun. 7, 10602 (2016).

8. Zhao, S., Osetsky, Y. N. \& Zhang, Y. Atomic-scale dynamics of edge dislocations in $\mathrm{Ni}$ and concentrated solid solution NiFe alloys. J. Alloy. Compd. 701, 1003-1008 (2017).

9. Rao, S. I. et al. Atomistic simulations of dislocations in a model BCC multicomponent concentrated solid solution alloy. Acta Mater. 125, 311-320 (2017).

10. Nöhring, W. G. \& Curtin, W. A. Dislocation cross-slip in fcc solid solution alloys. Acta Mater. 128, 135-148 (2017).

11. Yamakov, V., Wolf, D., Phillpot, S. R., Mukherjee, A. K. \& Gleiter, H. Deformationmechanism map for nanocrystalline metals by molecular-dynamics simulation. Nat. Mater. 3, 43-47 (2004).

12. Zaddach, A. J., Niu, C., Koch, C. C. \& Irving, D. L. Mechanical properties and stacking fault energies of NiFeCrCoMn high-entropy alloy. JOM 65, 1780-1789 (2013).

13. Zhao, S., Stocks, G. M. \& Zhang, Y. Stacking fault energies of face-centered cubic concentrated solid solution alloys. Acta Mater. 134, 334-345 (2017).

14. Huang, S. et al. Temperature dependent stacking fault energy of FeCrCoNiMn high entropy alloy. Scr. Mater. 108, 44-47 (2015).

15. Otto, F. et al. The influences of temperature and microstructure on the tensile properties of a CoCrFeMnNi high-entropy alloy. Acta Mater. 61, 5743-5755 (2013).

16. Smith, T. M. et al. Atomic-scale characterization and modeling of $60^{\circ}$ dislocations in a high-entropy alloy. Acta Mater. 110, 352-363 (2016).

17. Zhang, F. X. et al. Local structure and short-range order in a $\mathrm{NiCoCr}$ solid solution alloy. Phys. Rev. Lett. 118, 205501 (2017).
18. Li, Z. \& Raabe, D. Strong and ductile non-equiatomic high-entropy alloys: design, processing, microstructure, and mechanical properties. JOM 69, 2099-2106 (2017).

19. Bonny, G., Castin, N. \& Terentyev, D. Interatomic potential for studying ageing under irradiation in stainless steels: the FeNiCr model alloy. Model. Simul. Mater. Sci. Eng. 21, 85004 (2013).

20. Ogata, S., Li, J. \& Yip, S. Ideal pure shear strength of aluminum and copper Science 298, 807-811 (2002).

21. Shang, S. L. et al. Effects of alloying element and temperature on the stacking fault energies of dilute Ni-base superalloys. J. Phys. Condens. Matter 24, 505403 (2012).

22. Shang, S. L. et al. Generalized stacking fault energy, ideal strength and twinnability of dilute Mg-based alloys: a first-principles study of shear deformation. Acta Mater. 67, 168-180 (2014)

23. Bleskov, I., Hickel, T., Neugebauer, J. \& Ruban, A. Impact of local magnetism on stacking fault energies: a first-principles investigation for fcc iron. Phys. Rev. B 93 214115 (2016).

24. Qi, Y. \& Mishra, R. K. Ab initio study of the effect of solute atoms on the stacking fault energy in aluminum. Phys. Rev. B 75, 224105 (2007).

25. Eberhart, M. E. The metallic bond: elastic properties. Acta Mater. 44, 2495-2504 (1996).

26. Kioussis, N., Herbranson, M., Collins, E. \& Eberhart, M. E. Topology of electronic charge density and energetics of planar faults in fccmetals. Phys. Rev. Lett. 88 125501 (2002).

27. Rycroft, C. H. VORO++: a three-dimensional voronoi cell library in $\mathrm{C}++$. Chaos $\mathrm{An}$ Interdiscip. J. Nonlinear Sci. 19, 041111 (2009).

28. Zhao, S., Egami, T., Stocks, G. M. \& Zhang, Y. Effect of $d$ electrons on defect properties in equiatomic $\mathrm{NiCoCr}$ and $\mathrm{NiCoFeCr}$ concentrated solid solution alloys. Phys. Rev. Mater. 2, 013602 (2018).

29. Kresse, G. \& Furthmüller, J. Efficiency of ab-initio total energy calculations for metals and semiconductors using a plane-wave basis set. Comput. Mater. Sci. 6 , 15-50 (1996).

30. Perdew, J. P., Burke, K. \& Ernzerhof, M. Generalized gradient approximation made simple. Phys. Rev. Lett. 77, 3865 (1996).

31. Blöchl, P. E. Projector augmented-wave method. Phys. Rev. B 50, 17953 (1994).

32. Cowley, J. M. An approximate theory of order in alloys. Phys. Rev. 77, 669 (1950).

33. Cowley, J. M. Short-range order and long-range order parameters. Phys. Rev. 138 A1384 (1965).

34. Plimpton, S. Fast parallel algorithms for short-range molecular dynamics. J. Comput. Phys. 117, 1-19 (1995).

Open Access This article is licensed under a Creative Commons Attribution 4.0 International License, which permits use, sharing, adaptation, distribution and reproduction in any medium or format, as long as you give appropriate credit to the original author(s) and the source, provide a link to the Creative Commons license, and indicate if changes were made. The images or other third party material in this article are included in the article's Creative Commons license, unless indicated otherwise in a credit line to the material. If material is not included in the article's Creative Commons license and your intended use is not permitted by statutory regulation or exceeds the permitted use, you will need to obtain permission directly from the copyright holder. To view a copy of this license, visit http://creativecommons org/licenses/by/4.0/.

(c) The Author(s) 2019 